\title{
Gut permeability to lactulose and mannitol differs in treated Crohn's disease and celiac disease patients and healthy subjects
}

\author{
E.G. Vilela, H.O.G. Torres, M.L.A. Ferrari, A.S. Lima and A.S. Cunha \\ Instituto Alfa de Gastroenterologia, Hospital das Clínicas, Universidade Federal de Minas Gerais, Belo \\ Horizonte, MG, Brasil \\ Correspondence to: E.G. Vilela, Av. Professor Alfredo Balena, 189/803, 30130-100 Belo Horizonte, MG, \\ Brasil \\ Fax: +55-31-3274-6852. E-mail: evilela@medicina.ufmg.br
}

\begin{abstract}
The gut barrier monitors and protects the gastrointestinal tract from challenges such as microorganisms, toxins and proteins that could act as antigens. There is evidence that gut barrier dysfunction may act as a primary disease mechanism in intestinal disorders. The aim of the present study was to evaluate the barrier function towards sugars after the appropriate treatment of celiac disease and Crohn's disease patients and compare the results with those obtained with healthy subjects. Fifteen healthy volunteers, 22 celiac disease patients after 1 year of a gluten-free diet, and 31 Crohn's disease patients in remission were submitted to an intestinal permeability test with $6.0 \mathrm{~g}$ lactulose and $3.0 \mathrm{~g}$ mannitol. Six-hour urinary lactulose excretion in Crohn's disease patients was significantly higher than in both celiac disease patients $(0.42$ vs $0.15 \%)$ and healthy controls ( 0.42 vs $0.07 \%)$. Urinary lactulose excretion was significantly higher in celiac disease patients than in healthy controls $(0.15 \mathrm{vs} 0.07 \%)$. Urinary mannitol excretion in Crohn's disease patients was the same as healthy controls (21 vs $21 \%)$ and these values were significantly higher than in celiac disease patients (10.9\%). The lactulose/mannitol ratio was significantly higher in Crohn's disease patients in comparison to celiac disease patients $(0.021 \mathrm{vs} 0.013)$ and healthy controls $(0.021 \mathrm{vs} 0.003)$ and this ratio was also significantly higher in celiac disease patients compared to healthy controls (0.013 vs 0.003$)$. In spite of treatment, differences in sugar permeability were observed in both disease groups. These differences in the behavior of the sugar probes probably reflect different mechanisms for the alterations of intestinal permeability.
\end{abstract}

Key words: Intestinal permeability; Celiac disease; Crohn's disease; Mannitol; Lactulose

Received February 19, 2008. Accepted November 11, 2008

\section{Introduction}

The gastrointestinal tract is not only the primary organ of digestion and absorption, but it also constitutes the largest area of exposure to the outside environment in the human body. The function known as gut barrier is related to the integrity of the gastrointestinal tract and, at the same time, to the relationship between the microbiota of the intestinal lumen and the host. The concept of gut barrier is associated with permeability and is related to the property of a membrane that enables the passage of a solute by unmediated diffusion. On the other hand, absorption describes carriermediated modes of transportation of substances such as sodium and chloride across the intestine (1).
The development of methods for assessing intestinal barrier function became feasible after the introduction of non-metabolizable oligosaccharides as test substances in the 1970's (2). The oral administration of sugars and the subsequent measurement of these substances in the urine, denoted the intestinal permeability test, is a non-invasive method that has been used for the assessment of the integrity of the epithelial barrier.

There is evidence for the role of gut barrier dysfunction in intestinal disorders as a primary disease mechanism (3). Because of this, the intestinal permeability test is being used in both clinical practice and research.

The aim of the present study was to evaluate barrier function using the intestinal permeability test and to ana- 
lyze the behavior of individual probes after the adequate treatment of celiac disease patients, Crohn's disease patients in remission and compare the results between them and healthy controls.

\section{Patients and Methods}

Fifteen healthy volunteers ( 8 males, average age $36 \pm$ 6 years, range $23-46$ years) were submitted to the intestinal permeability test to establish control values.

Twenty-two celiac disease patients (11 males, average age $41 \pm 14$ years, range 19-74 years) who were diagnosed at least 1 year previously and were asymptomatic after 1 year of a gluten-free diet constituted the second group.

The third group was comprised of 31 Crohn's disease patients (18 males, average age $37 \pm 7$ years, range 19-54 years) in remission as defined by the Crohn's disease activity index (4). The mean index score was 56 (median 54). According to the Vienna Classification (5), the location of the disease was in the terminal ileum (L1) of 24 patients and in the ileocolon (L3) of 7 patients. The behavior was non-stricturing and non-penetrating (B1) in 5 patients, stricturing (B2) in 11 patients, and penetrating (B3) in 15 patients. Baseline medications were $1.0 \mathrm{~g}$ mesalazine three times/day for 10 patients, $150 \mathrm{mg}$ azathioprine once a day for 10 patients, azathioprine/mesalazine at the same doses for 2 patients, azathioprine/mesalazine plus $5 \mathrm{mg}$ a day prednisone in tapering doses for 4 patients, $50 \mathrm{mg}$ thalidomide twice a day for 1 patient, and thalidomide/ metronidazol (50 mg twice, $500 \mathrm{mg}$ three times a day, respectively) for 1 patient. Three patients were not using medication. All patients had more than 3 years of diagnosis and were in remission for more than 6 months. There were no patients whose remission was induced by surgery and the last surgical procedure was performed 6 years ago.

Celiac disease patients and Crohn's disease patients were seen at the outpatient clinic of the Instituto Alfa de Gastroenterologia, Hospital das Clínicas, Universidade Federal de Minas Gerais. Exclusion criteria were: patients younger than 18 years of age, pregnant or breast-feeding women, patients with renal failure, cirrhosis, congestive heart failure, nephrotic syndrome, diabetes and thyroid diseases that interfered with absorption, flux of water and solutes and intestinal motility in order to avoid interference with intestinal permeability tests (6)

In order to perform the intestinal permeability test, patients and healthy volunteers were seen at the hospital in the morning after a 10-h fast and were asked to eliminate residual urine. An isosmolar solution (120 mL) containing $6.0 \mathrm{~g}$ lactulose and $3.0 \mathrm{~g}$ mannitol was then ingested, and urine was collected into a sealed flask over a period of $6 \mathrm{~h}$. At the end of this period, $2.5 \mathrm{~mL}$ urine was stored in a second smaller flask and $0.6 \mathrm{mg}$ thimerosal was added to prevent bacterial growth. The samples were stored in liquid nitrogen.

Urinary lactulose and mannitol were analyzed by highperformance liquid chromatography (HPLC) using a Shimadzu $^{\circledR}$ system (Japan), which consisted of an injector pump, an auto-injector, a controller integrated with workstation software for interpretation of the results and a refractive index detector (6). For HPLC, urine was filtered through a micropore filter $(0.22 \mu \mathrm{L}$, Millex, Brazil), passed through an ion-exchange resin (Mixed-bed resin TMD-8, Sigma, USA), and then $50 \mu \mathrm{L}$ was injected into the chromatograph with the auto-injector. MilliQ water was used as mobile phase at a predetermined flow rate of $0.6 \mathrm{~mL} / \mathrm{min}$. A Supelcogel $33 \mathrm{H}^{\circledR}$ pre-column (USA) and a Rezek RHMmonosaccharide $\mathrm{H}^{+}(8 \%)^{\circledR}$ (USA) column were used for separation of the substances. In the refractive index module, different amplitudes of the waves generated by the sample containing lactulose and mannitol were captured and interpreted by the workstation, and the results were transformed into graphs. For standardization of the test and adequate interpretation of the data reported as $\mathrm{g} / \mathrm{L}$, an equation was generated using the areas under the curves calculated by the workstation and a line was drawn for the determination of the two substances (6). The results are reported in percentage of urinary excretion of both probes and the final result of the test is the ratio of administered test probes, since this is a more accurate indicator of permeation. Premucosal and postmucosal factors can influence the probes equally, and, therefore, the urinary excretion ratio shall not be affected (7).

Data showed a non-Gaussian distribution and are reported as medians. The non-parametric Mann-Whitney Utest was used for statistical analysis. The level of significance was set at $5 \%(P<0.05)$. All patients and controls freely signed an informed consent form, and the study was approved by the Ethics and Research Committee of the Universidade Federal de Minas Gerais.

\section{Results}

The descriptive analysis of data (median, 25 and 75 confidence interval and $P$ value of urinary lactulose and mannitol excretion and lactulose/mannitol ratio) is summarized in Table 1. Urinary lactulose excretion in Crohn's disease patients was significantly higher than in both celiac disease patients $(0.42$ vs $0.15 \%, P=0.001)$ and healthy controls $(0.42$ vs $0.07 \%, P=0.0001)$. When celiac disease patients were compared to healthy controls, the 
Table 1. Intestinal permeability of Crohn's disease and celiac disease patients for lactulose and mannitol.

\begin{tabular}{|c|c|c|c|c|c|c|}
\hline Groups & $\begin{array}{c}\text { \%Lactulose } \\
\text { [median (range)] }\end{array}$ & $P$ & $\begin{array}{c}\text { \%Mannitol } \\
\text { [median (range)] }\end{array}$ & $\mathrm{P}$ & $\begin{array}{l}\text { Lactulose/mannitol } \\
\text { [median (range)] }\end{array}$ & $P$ \\
\hline Crohn's disease & $0.42(0.15-0.99)$ & 0.001 & $21.0(13.5-29.5)$ & 0.0001 & $0.021(0.07-0.046)$ & 0.019 \\
\hline$v s$ & $v s$ & & $v s$ & & $v s$ & \\
\hline Celiac disease & $0.15(0.04-0.85)$ & & $10.9(3.3-19.5)$ & & $0.013(0.005-0.07)$ & \\
\hline Crohn's disease & $0.42(0.15-0.99)$ & 0.0001 & $21.0(13.5-29.5)$ & 0.54 & $0.021(0.07-0.046)$ & 0.001 \\
\hline vs & $v s$ & & vs & & $v s$ & \\
\hline Controls & $0.07(0.05-0.28)$ & & $21.0(18.3-28.0)$ & & $0.003(0.002-0.013)$ & \\
\hline Celiac disease & $0.15(0.04-0.85)$ & 0.039 & $10.9(3.3-19.5)$ & 0.001 & $0.013(0.005-0.07)$ & 0.001 \\
\hline$v s$ & $v s$ & & $v s$ & & $v s$ & \\
\hline Controls & $0.07(0.05-0.28)$ & & $21.0(18.3-28.0)$ & & $0.003(0.002-0.013)$ & \\
\hline
\end{tabular}

Subjects received $6.0 \mathrm{~g}$ lactulose and $3.0 \mathrm{~g}$ mannitol in $120 \mathrm{~mL}$ orally and a 6 -h urine was collected. Results are reported as percent of ingested sugar recovered in the 6-h urine. Crohn's disease, $\mathrm{N}=31$; celiac disease, $\mathrm{N}=22$; control, $\mathrm{N}=15$. The non-parametric Mann-Whitney U-test was used for statistical analysis.

urinary lactulose excretion was higher in the former $(0.15$ vs $0.07 \%, \mathrm{P}=0.039)$. Urinary mannitol excretion in Crohn's disease patients was the same as healthy controls (21 vs $21 \%, P=0.54)$ and these values were higher than in celiac disease patients $(10.9 \%, P=0.0001$ and $P=0.001$, respectively). Lactulose/mannitol ratio was significantly higher in Crohn's disease patients compared to celiac disease patients $(0.021$ vs $0.013, \mathrm{P}=0.019)$ and healthy controls $(0.021$ vs $0.003, P=0.001)$ and this ratio was also higher in celiac disease patients compared to healthy controls $(0.013$ vs $0.003, \mathrm{P}=0.001)$.

\section{Discussion}

The non-invasive functional intestinal permeability test is extremely important for both triage of active celiac disease, as well as for monitoring celiac patients on a glutenfree diet (8-10). It has been reported that celiac patients on adequate diet achieve normal intestinal permeability test results over the long term $(8,9)$. However, Bjarnason et al. (10), using chromium-labeled ethylenediaminetetraacetic acid ( ${ }^{51} \mathrm{Cr}$-EDTA), showed a persistent, but low-grade permeability alteration in celiac patients on a gluten-free diet. In Crohn's disease, there is a significant amount of evidence suggesting that increased permeability is an important factor in disease perpetuation and early alterations may reflect a change in the course of disease, before clinical manifestations (3). The intestinal permeability test may identify Crohn's disease patients in apparent remission, but with a high risk of clinical relapse $(1,3)$.

In spite of the existence of different theories to explain the mechanisms and pathways of intestinal permeability, it has been reported that reduced urinary mannitol excretion occurs in situations of villous hypotrophy $(7,11)$. Intestinal permeability of large molecules, such as lactulose, is not necessarily related to structural damage of the barrier, as has been shown with smaller molecules. Permeation ratios may be affected by intracellular mediators, such as cytokines, and inflammatory cells, such as neutrophils, known to be present in the mucosa of patients with disease of the small intestine (12). Cytokines, interferon- $\gamma$ and tumor necrosis factor (TNF), among others, cause tissue inflammation and increased sugar permeation by altering the resistance of tight junctions (13). The migration of neutrophils would also be related to the reduction of resistance of tight junctions (14). However, it is questionable whether such an alteration is an etiological factor or a consequence of the intestinal disease (15). Thus, it is reasonable to consider that pathological events may affect permeation of molecules in different ways: those related to villous hypotrophy will decrease urinary small molecule (i.e., mannitol) excretion and events associated with inflammation will cause an increase in urinary large molecule (i.e., lactulose) excretion, representing functional breakdown of the gut barrier and allowing antigen penetration.

Even though permeation rates of small and large probes are often described separately, greater emphasis is given to the relationship between the elimination of both probes, i.e., the lactulose and mannitol ratio (16-18). This is important because it avoids errors resulting from factors such as bacterial degradation of probes, which should affect both of them equally. On the other hand, since behavior of different probes may have different pathophysiological 
explanations, it might be interesting to look at each one in different diseases. Since intestinal permeability tests in the present group of patients were performed using the same methodology and around the same period of time and using the same control group, the differences observed in the behavior of probes may be of interest.

In the present study, it was observed that urinary lactulose excretion was increased and urinary mannitol excretion was decreased in celiac disease patients on a glutenfree diet compared with healthy controls. This means that, at some time, complete recovery of intestinal villous may not have occurred and an inflammatory process may have persisted (7). This continuing inflammation might be related to an eventual involuntary exposure of the intestinal mucosa to gliadin, with induction of an immunologic response that occurs both in the lamina propria and in the epithelium with a consequent reduction of resistance of tight junctions (19-23). The maintenance of intestinal permeability alterations in celiac disease patients is in agreement with the literature $(24,25)$.

In celiac disease patients, the most important mechanism proposed for deterioration of the gut barrier is associated with a reduction of intracellular expression of the molecule of epithelial cellular adhesion, named zonulin ZO-1, by gliadin (19). The immunological reaction that occurs in the presence of gliadin relies on the participation of CD4 lymphocytes and provides the opportunity, among other effects, for reduction in the expression of zonulin, which directly weakens the tight junctions and consequently increases intestinal permeability. If we take a look at the behavior of individual probes in celiac disease, alterations observed in this study are compatible with both villous atrophy, which is characteristic of celiac disease, and tight junction dysfunction related to gliadin effect through inflammation.

On the other hand, in a situation of clinical remission, alterations in the intestinal permeability test may represent an early functional change in the intestinal mucosa that could indicate early relapses of Crohn's disease, or even initiate them (26-28). An alteration in the gut barrier may lead to the absorption of endotoxin and lipopolysaccharides from the lumen. Both of these substances are potent stimulators of acute-phase reactions and of secretion of interleukin-6 and TNF, which have been shown to be important mediators of inflammation in Crohn's disease (29). When results are analyzed, alterations in the gut barrier, as expressed by lactulose/mannitol ratio, are higher in Crohn's disease patients than in celiac disease patients, mainly due to higher lactulose values, since mannitol excretion rates are similar to controls. This is compatible with normal villous morphology in these patients, whose disease is in remission and in whom we may suppose villous are preserved. It can also be supposed that a inflammatory reaction that is characteristic of the disease may result in a weakness of the tight junctions. There is no data in the literature regarding recovery of gut barrier function after use of baseline medications.

In short, even though the lactulose/mannitol relationship is the most reliable indicator of intestinal permeability changes because it approaches pre- and post-mucosal factors that may affect small and large molecules equally, the observation of the isolated behavior of specific probes might disclose different mechanisms that could be important in the study of intestinal disease. Also, in spite of treatment and remission, persistent alterations were seen in both groups of disease demonstrating subtle changes that can be identified by intestinal permeability tests.

of Gastroenterology, Vienna 1998. Inflamm Bowel Dis 2000; 6: 8-15.

6. Garcia Vilela E, De Lourdes De Abreu Ferrari M, Oswaldo Da Gama TH, Guerra Pinto A, Carolina Carneiro Aguirre A, Paiva Martins F, et al. Influence of Saccharomyces boulardii on the intestinal permeability of patients with Crohn's disease in remission. Scand J Gastroenterol 2008; 43: 842848.

7. Travis S, Menzies I. Intestinal permeability: functional assessment and significance. Clin Sci 1992; 82: 471-488.

8. Vogelsang $\mathrm{H}$, Schwarzenhofer $\mathrm{M}$, Oberhuber $\mathrm{G}$. Changes in gastrointestinal permeability in celiac disease. Dig Dis 1998; 16: 333-336.

9. Perry I, Iqbal T, Cooper B. Intestinal permeability in coeliac disease. Lancet 2001; 358: 1729-1730. 
10. Bjarnason I, Peters TJ, Veall N. A persistent defect in intestinal permeability in coeliac disease demonstrated by a 51Cr-labelled EDTA absorption test. Lancet 1983; 1: 323325.

11. Menzies IS, Laker MF, Pounder R, Bull J, Heyer S, Wheeler $P G$, et al. Abnormal intestinal permeability to sugars in villous atrophy. Lancet 1979; 2: 1107-1109.

12. Hollander D. The intestinal permeability barrier. A hypothesis as to its regulation and involvement in Crohn's disease. Scand J Gastroenterol 1992; 27: 721-726.

13. Nash S, Stafford J, Madara JL. The selective and superoxide-independent disruption of intestinal epithelial tight junctions during leukocyte transmigration. Lab Invest 1988; 59: 531-537.

14. Brown SJ, Mayer L. The immune response in inflammatory bowel disease. Am J Gastroenterol 2007; 102: 2058-2069.

15. Hollander D. Crohn's disease, TNF-alpha, and the leaky gut. The chicken or the egg? Am J Gastroenterol 2002; 97: 1867-1868.

16. van Elburg RM, Uil JJ, Mulder CJ, Heymans HS. Intestinal permeability in patients with coeliac disease and relatives of patients with coeliac disease. Gut 1993; 34: 354-357.

17. Teahon K, Somasundaram S, Smith T, Menzies I, Bjarnason I. Assessing the site of increased intestinal permeability in coeliac and inflammatory bowel disease. Gut 1996; 38: 864-869.

18. Secondulfo M, de Magistris L, Fiandra R, Caserta L, Belletta M, Tartaglione MT, et al. Intestinal permeability in Crohn's disease patients and their first degree relatives. Dig Liver Dis 2001; 33: 680-685.

19. Fasano A, Not T, Wang W, Uzzau S, Berti I, Tommasini A, et al. Zonulin, a newly discovered modulator of intestinal permeability, and its expression in coeliac disease. Lancet 2000; 355: 1518-1519.
20. Uil JJ, van Elburg RM, van Overbeek FM, Meyer JW, Mulder CJ, Heymans HS. Follow-up of treated coeliac patients: sugar absorption test and intestinal biopsies compared. Eur J Gastroenterol Hepatol 1996; 8: 219-223.

21. Duerksen DR, Wilhelm-Boyles C, Parry DM. Intestinal permeability in long-term follow-up of patients with celiac disease on a gluten-free diet. Dig Dis Sci 2005; 50: 785-790.

22. Vilela EG, de Abreu Ferrari ML, de Gama Torres HO, Martins FP, Goulart EM, Lima AS, et al. Intestinal permeability and antigliadin antibody test for monitoring adult patients with celiac disease. Dig Dis Sci 2007; 52: 1304-1309.

23. Arrieta MC, Bistritz L, Meddings JB. Alterations in intestinal permeability. Gut 2006; 55: 1512-1520.

24. Johnston SD, Smye M, Watson RG. Intestinal permeability and morphometric recovery in coeliac disease. Lancet 2001; 358: 259-260.

25. Bjarnason I, Peters TJ. In vitro determination of small intestinal permeability: demonstration of a persistent defect in patients with coeliac disease. Gut 1984; 25: 145-150.

26. Wyatt $J$, Vogelsang $H$, Hubl $W$, Waldhoer $T$, Lochs $H$. Intestinal permeability and the prediction of relapse in Crohn's disease. Lancet 1993; 341: 1437-1439.

27. D'Inca R, Di Leo V, Corrao G, Martines D, D'Odorico A, Mestriner $C$, et al. Intestinal permeability test as a predictor of clinical course in Crohn's disease. Am J Gastroenterol 1999; 94: 2956-2960.

28. Meddings JB. Review article: Intestinal permeability in Crohn's disease. Aliment Pharmacol Ther 1997; 11 (Suppl 3): 47-53.

29. Li Q, Zhang Q, Wang M, Zhao S, Ma J, Luo N, et al. Interferon-gamma and tumor necrosis factor-alpha disrupt epithelial barrier function by altering lipid composition in membrane microdomains of tight junction. Clin Immunol 2008; 126: 67-80. 